\title{
Drug Resistance Patterns of Bacterial Pathogens from Adult Patients with Pneumonia in Arba Minch Hospital, South Ethiopia
}

\section{Belayneh Regasa*}

Department of Medical Microbiology, Arba Minch University, Arba Minch, Ethiopia

"Corresponding author: Belayneh Regasa, Department of Medical Microbiology, Arba Minch University, Arba Minch, Ethiopia, Tel: 251468810771; E-mail: belayjanimen@gmail.com

Rec Date: May 06, 2014, Acc date: August 27, 2014, Pub date: August 29, 2014

Copyright: (C) 2014 Regasa B. This is an open-access article distributed under the terms of the Creative Commons Attribution License, which permits unrestricted use, distribution, and reproduction in any medium, provided the original author and source are credited.

\begin{abstract}
Background: Community-acquired pneumonia (CAP) is associated with high mortality. Drug resistance is common in countries where the alternative treatments are limited and available drugs are misused. In resource limited countries like Ethiopia; it is wise to determine antimicrobial susceptibility pattern of common bacterial pathogens of CAP. The objective of our study was to determine antimicrobial susceptibility pattern of common bacterial pathogens of CAP among adult patients visiting Arba Minch Hospital.

Methods: A cross sectional study conducted at Arba Minch Hospital, Southern Ethiopia from February to May 2013. Sputum specimens were collected; microbiological investigations and antimicrobial susceptibility testing were performed using standard procedures. Data was processed and analyzed with SPSS version16.0. Results: Out of 170 cases, only $73(42.9 \%)$ were culture positive. Majority of tested bacterial isolates $(>86 \%)$ were sensitive to Ceftriaxone and Ciprofloxacin. Most S. pneumoniae isolates (60\%) were resistant to Oxacillin. Most of S. aureus and gram negative bacterial isolates were resistance to Tetracycline (100\%), Penicillin (83.3\%), Ampicillin (50-100\%), Doxycycline (50-100\%), and Trimethoprim-sulfamethoxazole (83.3-100\%). Multidrug resistance (MDR) was observed to most $(60.3 \%)$ bacterial isolates.
\end{abstract}

Conclusion: Antimicrobial resistance including MDR was observed to a number of commonly used antibiotics, such as trimethoprim- sulfamethoxazole, penicillin groups and doxycycline. Hence, periodic monitoring of drug resistant pattern is essential for better management of CAP.

Keywords: CAP; Bacterial pathogens; Antimicrobial susceptibility pattern

\section{Introduction}

Community-acquired pneumonia (CAP) affects 3-5 adults per thousand per year with a mortality of $7-14 \%$ in hospitalized patients [1]. It is associated with high mortality. About 5.6 million cases of CAP are reported in the United States each year, with an associated mortality rate of approximately $14 \%[2,3]$. Despite the advent of potent antibiotic over the last decades, significant mortality is still associated with CAP [4]. Increased antibiotic resistance in frequently isolated bacterial pathogens from CAP patients has complicated the selection process of antimicrobial agents [5] and the clinical presentation is usually not specific enough to make a firm etiologic diagnosis [6]. The resistant strains of bacteria can quickly multiply and spread within a community where antibiotic use is common. Consequently, antibiotic resistance often results in various societal costs, including increased drug costs, additional health-service costs (such as laboratory tests and hospitalizations)and greater drug resistance-related morbidity and mortality, and productivity losses [7]. In resource limited developing countries like Ethiopia; it is wise to determine antimicrobial susceptibility pattern of bacterial pathogens. This might help for the management of the case in case of emergency and helps for the rational utilization of antimicrobial agents.

\section{Methods and Materials}

During the period February to May 2013 a total of 170 adults (above 15 years old) with typical symptoms of the disease, such as productive cough, fever, chest pain and the presence of consolidate on the chest radiograph consistent with pneumonia was included in this study. Sputum samples were inoculated onto Blood, Mac Conkey, Manitol Salt agar (MSA) and Chocolate agar (Oxoid Ltd, UK) plates [8]. The bacterial isolates were then identified and subjected to antimicrobial susceptibility testing according to Clinical Laboratory Standards Institute (CLSI) recommendations [9,10]. The antibiotic discs used and their concentration were:- Ceftriaxone (CRO, $30 \mu \mathrm{g})$, Ciprofloxacin (CIP, $5 \mu \mathrm{g}$ ), Tetracycline (TE, $30 \mu \mathrm{g}$ ), Chloramphenicol $(\mathrm{C}, 30 \mu \mathrm{g})$, Erythromycin (E, $15 \mu \mathrm{g})$, Doxycycline (DO, $30 \mu \mathrm{g})$, Penicillin ( $\mathrm{P}, 10 \mu \mathrm{g})$, Gentamycin (CN, $10 \mu \mathrm{g}$ ), Trimethoprimsulfamethoxazole (TMP-SMX, 1.25+23.75 $\mu \mathrm{g}$ ), Ampicillin (AMP, 10 $\mu \mathrm{g})$ and Oxacillin (OXA, $1 \mu \mathrm{g}$ ) All antibiotic were obtained from Oxoid Limited, Basingstoke Hampshire, UK. A standard inoculum adjusted to 0.5 McFarland was swabbed on to Muller-Hinton agar (Oxoid Ltd. Bashingstore Hampaire, UK); antibiotic disc were dispensed after drying the plate for $3-5 \mathrm{~min}$ and incubated at $37^{\circ} \mathrm{C}$ for 24 hours. For $S$. pneumoniae, MHA supplied with 5\% sheep blood and for $H$. influenzae, MHA chocolate agar was used. Quality control strains that were used include: Staphylococcus aureus ATCC 25923, Escherichia coli ATCC 25922, and Pseudomonas aeruginosa ATCC 27853 [10]. Selected Socio-demographic characteristics like age and sex were obtained. Data were entered and analyzed using SPSS version 
Page 2 of 4

16.0 computer software.The proposal of this study was ethically approved by the Institutional Ethical Review Committee (IRC) of Arba Minch University. Permission was obtained from Medical director of Arba Minch Hospital. Written informed consent was obtained from each patient participated in the study.

\section{Result}

A total of 170 adult patients clinically diagnosed to have CAP in Arba Minch Hospital were selected and participated in this study (Table 1). Of these, $95(55.9 \%)$ were males and 75 (44.1\%) were females.

The isolated bacteria were, Streptococcus pneumoniae 20 (11.8\%), Staphylococcus aureus 18 (10.6\%), Pseudomonas aeruginosa 12 (7.1\%), Klebsiella pneumoniae 11 (6.5\%), Escherichia coli 5 (2.9\%), Proteus mirabilis 2 (1.2\%), Proteus vulgaris 1 (0.6\%) and Haemophilus influenzae $4(2.4 \%)$.

\begin{tabular}{|l|l|l|}
\hline \multicolumn{2}{|c|}{ Variables } & Number (\%) \\
\hline \multirow{3}{*}{ Sex } & Male & $95(55.9)$ \\
\cline { 2 - 3 } & Female & $75(44.1)$ \\
\hline \multirow{3}{*}{ Age } & $15-25$ & $23(13.5)$ \\
\cline { 2 - 3 } & $26-45$ & $67(39.5)$ \\
\cline { 2 - 3 } & $46-65$ & $63(37)$ \\
\cline { 2 - 3 } & $>65$ & $17(10)$ \\
\hline
\end{tabular}

Table 1: Distribution of community-acquired pneumonia by sex and age $(n=170)$

\begin{tabular}{|c|c|c|c|c|c|c|c|c|c|c|c|c|}
\hline \multirow[t]{2}{*}{$\begin{array}{l}\text { Bacterial } \\
\text { isolates }\end{array}$} & \multicolumn{12}{|c|}{$\begin{array}{l}\text { Drugs tested } \\
\text { No(\%) resistance }\end{array}$} \\
\hline & No & $\mathrm{CRO}$ & CIP & TE & C & E & DO & $P$ & $\mathrm{CN}$ & TMP-STX & AMP & OXA \\
\hline S. pneumoniae & 20 & NA & NA & $10(50)$ & $1(5)$ & $1(5)$ & NA & NA & NA & $1(5)$ & NA & $12(60)$ \\
\hline S. aureus & 18 & $4(22.2)$ & $4(22.2)$ & $18(100)$ & $5(27.8)$ & $9(50)$ & $9(50)$ & $15(83.3)$ & $5(27.8)$ & $15(83.3)$ & 15 (83.3) & $15(83.3)$ \\
\hline P. aeruginosa & 12 & $1(8.3)$ & $1(8.3)$ & NA & NA & NA & NA & NA & $7(58.3)$ & NA & NA & NA \\
\hline K. pneumoniae & 11 & 0 & 0 & $11(100)$ & $2(18.2)$ & NA & $1(9.1)$ & NA & $2(18.2)$ & $11(100)$ & $11(100)$ & NA \\
\hline P. mirabilis & 2 & $1(50)$ & $1(50)$ & $2(100)$ & $2(100)$ & NA & $2(100)$ & NA & $2(100)$ & $2(100)$ & $2(100)$ & NA \\
\hline P. vulgaris & 1 & 0 & 0 & 1 & 1 & NA & 1 & NA & 1 & 1 & 1 & NA \\
\hline E. coli & 5 & 0 & 0 & $5(100)$ & $5(100)$ & NA & $5(100)$ & NA & $1(20)$ & $5(100)$ & $5(100)$ & NA \\
\hline H. influenzae & 4 & $1(25)$ & $1(25)$ & $4(100)$ & $1(25)$ & NA & NA & NA & NA & $4(100)$ & $2(50)$ & NA \\
\hline Total & 73 & $7(13.2)$ & $7(13.2)$ & $51(83.6)$ & $18(29.5)$ & $10(26.3)$ & $18(48.6)$ & $15(83.3)$ & $18(36.7)$ & 39 (63.9) & $36(87.8)$ & $27(71.1)$ \\
\hline
\end{tabular}

Table 2: Drug resistance pattern of bacterial isolates from adult patients with Community-acquired pneumonia in Arba Minch Hospital, 2013

Streptococcus pneumoniae isolates showed relatively high resistance (60\%) to Oxacillin (penicillin group representative) and all isolates were sensitive against Trimethoprim-sulfamethoxazole. High resistance rate $S$. aureus was observed to Tetracycline (100\%), Oxacillin (83.3\%), Ampicillin (83.3\%), Penicillin (83.3\%), Trimethoprim-sulfamethoxazole (83.3\%), Erythromycin (50\%) and Doxycycline (50\%). Pseudomonas aeruginosa isolates showed that $50 \%$ resistant to Gentamycin. The antimicrobial testing of $K$. pneumoniae and $H$. influenzae isolates indicated that all isolates showed resistance (100\%) to Tetracycline, Ampicillin and Trimethoprim-sulfamethoxazole. Proteus and E. coli isolates showed resistance to Tetracycline, Chloramphenicol, Doxycycline, Gentamycin, Ampicillin and Trimethoprim-sulfamethoxazole (Table 2).

Multidrug resistance was also observed to a number of antimicrobial agents (Table 3)

\section{Discussion}

The importance of knowing susceptibility patterns of bacterial isolates in patients with Community-acquired pneumonia has been identified as a key step towards limiting unnecessary antibacterial prescribing and treating patients effectively, which was the main purpose of this study.

S. pneumoniae, which was the commonest isolate in this study, showed $60 \%$ resistant to oxacillin which is representative to penicillin group. This finding is comparable to studies conducted in USA (53\%) [11] and Iran (30-57\%) [12]. In this study, most tested $S$. pneumoniae isolates showed that $95 \%$ susceptible to trimethoprimsulfamethoxazole, but studies conducted in Nigeria (100\%) [13] and Kenya (54\%) [14], showed high resistance rate of $S$. pneumoniae to trimethoprim-sulfamethoxazole. In addition, $95 \%$ of tested $\mathrm{S}$. pneumoniae isolates were susceptible to chloramphenicol and erythromycin. These findings are comparable to a study conducted in Kenya (>97\%) [14]. 


\begin{tabular}{|c|c|c|}
\hline Bacterial Isolates & Resistance Antibiogram & No $(\%)$ \\
\hline S. pneumoniae & OXA, TE & $2(10)$ \\
\hline$(n=20)$ & OXA, TE, C, E & $1(5)$ \\
\hline \multirow{10}{*}{ S. aureus $(n=18)$} & OXA, TE, P, AMP & $2(12.5)$ \\
\hline & OXA, AMP, E, DO, TMP-STX & $1(6.3)$ \\
\hline & P, TE, E, DO, TMP-STX & $1(6.3)$ \\
\hline & OXA, AMP, TE, E, DO, TMP-STX & $2(12.5)$ \\
\hline & OXA, AMP, P, TE, E, TMP-STX & $1(6.3)$ \\
\hline & OXA, AMP, P, TE, DO, TMP-STX & $1(6.3)$ \\
\hline & OXA, AMP, P, TE, DO, E, TMP-STX & $1(6.3)$ \\
\hline & OXA, AMP, P, TE, DO, C, E, CIP, TMP-STX & $1(6.3)$ \\
\hline & OXA, AMP, P, TE, C, E, CN, CRO, TMP-STX & $2(12.5)$ \\
\hline & $\begin{array}{l}\text { OXA, AMP, P, TE, C, E, CN, CRO, CIP, TMP- } \\
\text { STX }\end{array}$ & $4(25)$ \\
\hline P. aeruginosa & $\mathrm{CN}, \mathrm{CRO}$ & $2(20)$ \\
\hline$(n=12)$ & $\mathrm{CN}, \mathrm{CRO}, \mathrm{CIP}$ & $2(20)$ \\
\hline K. pneumoniae & AMP, TE, TMP-STX & $5(45.5)$ \\
\hline \multirow[t]{4}{*}{$(n=11)$} & AMP, TE, CRO, TMP-STX & $2(18.9)$ \\
\hline & AMP, TE, CN, TMP-STX & $2(18.9)$ \\
\hline & AMP, TE, C, TMP-STX & $1(12.5)$ \\
\hline & AMP, TE, CN, CRO, TMP-STX & $1(12.5)$ \\
\hline \multirow{2}{*}{ P. mirabilis $(\mathrm{n}=2)$} & AMP, TE, DO, C, CN, TMP-STX & $1(50)$ \\
\hline & AMP, TE, DO, C, CN, CRO, CIP, TMP-STX & $1(50)$ \\
\hline$P$. vulgaris $(\mathrm{n}=1)$ & AMP, TE, DO, C, TMP-STX & $1(100)$ \\
\hline \multirow{2}{*}{ E. coli $(n=5)$} & AMP, TE, DO, C, TMP-STX & $3(60)$ \\
\hline & AMP, TE, DO, C, CN, TMP-STX & $2(40)$ \\
\hline \multirow{2}{*}{ H. influenzae $(\mathrm{n}=4)$} & AMP, TE, TMP-STX & $1(33.3)$ \\
\hline & AMP, TE, C, CIP, TMP-STX & $1(33.3)$ \\
\hline Total $(n=73)$ & & $44(60.3)$ \\
\hline
\end{tabular}

Table 3: Multi-drug resistance Antibiogram of bacterial isolates from adult patients with Community-acquired pneumonia in Arba Minch Hospital, 2013

The second most causative agent $S$. aureus showed $77.8 \%$ susceptible to ceftriaxone and ciprofloxacin, and $72.2 \%$ to gentamycin and chloramphenicol. This result is comparable to studies conducted in Ibadan, Nigeria (66.7\% ciprofloxacin and $66.7 \%$ gentamycin) [13] and Benin City, Nigeria (66.7\% ceftriaxone, $66.7 \%$ ciprofloxacin, and $66.7 \%$ chloramphenicol) [15]. In addition $83.3 \%$ of tested $S$. aureus showed resistance to penicillin, ampicillin, oxacillin and trimethoprim-sulfamethoxazole; which is comparable to studies conducted China (88.7\% resistance to penicillin) [16] and Nigeria (resistance rate of $66.7 \%$ for penicillin) [13], but lower than study conducted in Nigeria (100\% for trimethoprim-sulfamethoxazole) [13].

Most of tested gram negative bacilli isolates were sensitive (90\%) to ceftriaxone and ciprofloxacin. These findings are comparable to studies conducted in Benin City, Nigeria (66-100\%) [15] and Ibadan, Nigeria (60-100\%) [13]. Majority of gram negative bacilli was resistance $(100 \%)$ to tetracycline, chloramphenicol, doxycycline (except K. Pneumoniae, 90\% susceptible), trimethoprimsulfamethoxazole and ampicillin. Similar study conducted in Nigeria (60-100\%) [15], supports these findings. The commonest causative agent among gram negative bacilli, $P$. aeruginosa, showed $58.3 \%$ resistance to gentamycin, which is comparable to study conducted in Nigeria (53.6\%) [13]. However, it showed low resistance (8.3\%) to ceftriaxone and ciprofloxacin; but study conducted in Nigeria $(39.3 \%$ resistance for ciprofloxacin) [13], showed high resistance. $K$. pneumoniae and E. coli showed $100 \%$ resistance to tetracycline, ampicillin and trimethoprim-sulfamethoxazole. These findings are comparable to studies conducted in Benin City, Nigeria (100\% resistance to tetracycline) [15] and Ibadan, Nigeria (100\% resistance to trimethoprim-sulfamethoxazole) [13]. All tested Proteus species isolates were resistance (100\%) to doxycycline, tetracycline, and ampicillin and trimethoprim-sulfamethoxazole. These findings are comparable to study conducted in Ibadan, Nigeria (100\% resistance to trimethoprim-sulfamethoxazole) [13].

All $H$. influenzae isolates tested for antimicrobial sensitivity showed low resistance (25\%) to ceftriaxone, ciprofloxacin and chloramphenicol. These findings are comparable to study conducted in Nigeria (chloramphenicol 30.3\% and ciprofloxacin 26.1\%) [13]. In most of tested $H$. influenzae isolates, high resistance rate to tetracycline (100\%), ampicillin (50\%) and trimethoprimsulfamethoxazole $(100 \%)$ were observed. These findings are similar with studies conducted in USA (47\% resistance to ampicillin) [17] and Nigeria (93.7\% resistance to trimethoprim-sulfamethoxazole) [13], but is not as high as that observed in other countries such as in China (>90\% susceptibility to most antibiotics) [16]. The differences in antibiotic resistance patterns may be due to variations in the antibiotic prescribing habits in different geographical regions.

\section{Conclusion}

In the present study, most bacterial isolates were susceptible to ceftriaxone and ciprofloxacin. However, antimicrobial resistance including Multidrug resistance was observed to a number of commonly used antibiotics, such as trimethoprim-sulfamethoxazole, penicillin group and doxycycline. Hence, it is important to periodically monitor the antibiotic resistance patterns to aid physicians to choose empirical treatments for better management of CAP.

\section{Acknowledgements}

The author would like to thank those who were involved in this research.

\section{References}

1. Schouten JA, Hulscher ME, Kullberg BJ, Cox A, Gyssens IC, et al. (2005) Understanding variation in quality of antibiotic use for communityacquired pneumonia: effect of patient, professional and hospital factors. J Antimicrob Chemother 56: 575-582. 
Citation: Regasa B. (2014) Drug Resistance Patterns of Bacterial Pathogens from Adult Patients with Pneumonia in Arba Minch Hospital, South Ethiopia. J Med Microb Diagn 3: 151. doi:10.4172/2161-0703.1000151

Page 4 of 4

2. Bosker G (2002) Community-acquired pneumonia (CAP) antibiotic selection and management update. Hospital Medicine Consensus Reports: $1-32$

3. Gleason PP (2002) The emerging role of atypical pathogens in community-acquired pneumonia. Pharmacotherapy 22: 2S-11S.

4. Hui KP, Chin NK, Chow K, Brownlee A, Yeo TC, et al. (1993) Prospective study of the aetiology of adult community acquired bacterial pneumonia needing hospitalisation in Singapore. Singapore Med J 34: 329-334.

5. Guthrie R (2001) Community-acquired lower respiratory tract infections: etiology and treatment. Chest 120: 2021-2034.

6. Okesola AO, Ige OM (2008) Trends in bacterial pathogens of lower respiratory tract infections. Indian J Chest Dis Allied Sci 50: 269-272.

7. Lim YW, Steinhoff M, Girosi F, Holtzman D, Campbell H, et al. (2006) Reducing the global burden of acute lower respiratory infections in children: the contribution of new diagnostics. Nature 444 Suppl 1: 9-18.

8. Mohammed E, Muhe L, Geyid A (2004) Prevalence of acute respiratory bacterial pathogens in children in Gondar. Ethiop J Heath Dev 14: 191-97

9. Cheesbrough M (2006) District Laboratory Practice in Tropical Countries. Part 2. 2nd ed. Cambridge University: 76-79 and 132-143

10. National Committee for Clinical Laboratory Standards (NCCLS) (2010) Performance Standards for antimicrobial susceptibility testing. 20th Informational Supplement. M100-S20 (ISBN 1-56238- 716 -2). Clinical and Laboratory Standards Institute. Pennsylvania. USA.

11. Selman LJ, Mayfield DC, Thornsberry C, (2000) Changes in single- and multiple-drug resistance among Streptococcus pneumoniae over three years (1997-2000) [abstract 1789]. In: Program and abstracts of the 40th Interscience Conference on Antimicrobial Agents and Chemotherapy (Toronto, Canada). Washington, DC.

12. Hashemi SH, Soozanchi G, Jamal-Omidi S, Yousefi-Mashouf R, Mamani $\mathrm{M}$, et al. (2010) Bacterial aetiology and antimicrobial resistance of community-acquired pneumonia in the elderly and younger adults. Trop Doct 40: 89-91.

13. Okesola AO, Ige OM (2008) Trends in bacterial pathogens of lower respiratory tract infections. Indian J Chest Dis Allied Sci 50: 269-272.

14. Scott JA, Hall AJ, Muyodi C, Lowe B, Ross M, et al. (2000) Aetiology, outcome, and risk factors for mortality among adults with acute pneumonia in Kenya. Lancet 355: 1225-1230.

15. Egbagbe EE, Mordi RM (2006) Aetiology of Lower Respiratory Tract Infection in Benin City, Nigeria. JMBR 5: 22-27

16. Wang H, Liu YL, Chen MJ, Xu YC, Sun HL, et al. (2012) Antimicrobial susceptibility of community-acquired respiratory tract pathogens isolated from adults in China during 2009 and 2010. ZhonghuaJie $\mathrm{He} \mathrm{He} \mathrm{Hu} \mathrm{Xi}$ ZaZhi 35: 113-119.

17. Doern GV, Jones RN, Pfaller MA (1999) Haemophilusinfluenzae and Moraxella catarrhalis from patients with community-acquired respiratory tract infections: antimicrobial susceptibility patterns from the SENTRY Antimicrobial Surveillance Program.Antimicrob Agents Chemother 43: 385-389. 